University of Nebraska - Lincoln

DigitalCommons@University of Nebraska - Lincoln

Ralph Skomski Publications

Research Papers in Physics and Astronomy

2-28-2008

\title{
Temperature- and field-induced entropy changes in nanomagnets
}

\author{
Ralph Skomski \\ University of Nebraska-Lincoln, rskomski2@unl.edu \\ Christian Binek \\ University of Nebraska, Lincoln, cbinek@unl.edu \\ Tathagata Mukherjee \\ University of Nebraska-Lincoln, tatha.muk@gmail.com \\ Sarbeswar Sahoo \\ ssahoo2@unl.edu \\ David J. Sellmyer \\ University of Nebraska-Lincoln, dsellmyer@unl.edu
}

Follow this and additional works at: https://digitalcommons.unl.edu/physicsskomski

Part of the Physics Commons

Skomski, Ralph; Binek, Christian; Mukherjee, Tathagata; Sahoo, Sarbeswar; and Sellmyer, David J., "Temperature- and field-induced entropy changes in nanomagnets" (2008). Ralph Skomski Publications. 53.

https://digitalcommons.unl.edu/physicsskomski/53

This Article is brought to you for free and open access by the Research Papers in Physics and Astronomy at DigitalCommons@University of Nebraska - Lincoln. It has been accepted for inclusion in Ralph Skomski Publications by an authorized administrator of DigitalCommons@University of Nebraska - Lincoln. 


\title{
Temperature- and field-induced entropy changes in nanomagnets
}

\author{
Ralph Skomski, ${ }^{\text {a) }}$ Christian Binek, T. Mukherjee, S. Sahoo, and D. J. Sellmyer \\ Department of Physics and Astronomy and Nebraska Center for Materials and Nanoscience, \\ University of Nebraska, Lincoln, Nebraska 68588, USA
}

(Presented on 6 November 2007; received 11 September 2007; accepted 1 November 2007; published online 28 February 2008)

\begin{abstract}
Room-temperature magnetic-entropy changes in nanostructures for magnetic refrigeration are investigated by model calculations. Using a mean-field approach, the magnetic entropy is calculated as a function of temperature, magnetic field, particle size, anisotropy, and interaction strength. Both isotropic (Heisenberg) and uniaxial (Ising and $X Y$ ) anisotropies are considered. The nanoparticle entropy strongly depends on the character of the anisotropy, in contrast to atomic ferromagnetism, where the anisotropy energy is much smaller than the interaction energy. Most promising are isotropic particles and particles with weak easy axis anisotropy, as well as easy-plane particle with the field in the plane. The optimum nanoparticle size is not much larger than $1 \mathrm{~nm}$, because the relative magnetization direction in a nanoparticle is usually frozen and do not contribute to the entropy change. (C) 2008 American Institute of Physics. [DOI: 10.1063/1.2835094]
\end{abstract}

Magnetic refrigeration is an energy-efficient and environment-friendly technology. First, magnetic refrigeration does not use ozone-depleting chemicals and is therefore environmentally friendly. Second, the cooling efficiency is better than that of gas-compression refrigeration. For many decades, a similar idea has been applied in low temperature physics, in the adiabatic demagnetization of paramagnetic salts, but advanced research focuses on magnetic refrigeration at room temperature. ${ }^{1-3}$ Present-day magnetic cooling relies on atomic degrees of freedom, such as the spin orientation of Gd $4 f$ shells in magnetic compounds. Most fundamental and applied research has focused on bulk materials such as $\mathrm{Gd}_{3} \mathrm{Ge}_{2} \mathrm{Si}_{2}$, ${ }^{1,2}$ which can be produced in large amounts. However, the number of suitable compounds is limited. Almost all materials show some magnetocaloric effect, but the entropy gain is typically small and appreciable only in inconvenient field and temperature regions. Nanostructures are far more flexible with respect to the optimization of material properties, including magnetocaloric effects. $^{3-5}$ One advantage is that the magnetization of suitable nanostructures can be switched by an applied magnetic field of the order one $1 \mathrm{~T}(10 \mathrm{kOe})$ or less, which are easily realized by using permanent magnets such as $\mathrm{Nd}_{2} \mathrm{Fe}_{14} \mathrm{~B}$ and do not require superconducting magnets. 6,7

The idea behind magnetic cooling is to exploit entropy changes in spin systems. The starting point is the free energy

$$
F=U-T S-\mu_{0} \int \mathbf{M} \cdot \mathbf{H} d V
$$

where $U$ is the internal energy, $T$ is the temperature, $M$ is the magnetization, and $H$ is the magnetic field. The entropy exploited in magnetic cooling originates from the relative orientation of the atomic magnetic moments, meaning that a field changes the entropy $S$ and, via $M$ and $T S$, the temperature. While a magnetic field aligns the spin and thereby re-

${ }^{a)}$ Electronic mail: rskomski@neb.rr.com. duces the entropy, only a part of Zeeman energy $\mu_{0} \int \mathbf{M} \cdot \mathbf{H}$ $d V$ enters the magnetic entropy balance. Let us consider nanoparticles containing $N$ spins of moment $\mu_{B}(\operatorname{spin} 1 / 2)$, or $N / S_{\text {at }}$ atoms, where $S_{\text {at }}$ is the number of spins per atom ( $S_{\mathrm{at}}=7 / 2$ for $\mathrm{Gd}^{3+}$ ). Figure 1 compares the Curie transition (a) with a Stoner-Wohlfarth-type micromagnetic magnetization rotation (b), as typically encountered in small particles. Furthermore, one must keep in mind that magnetization changes in nanostructures are often micromagnetic, that is, unrelated to thermal fluctuations and not accompanied by entropy changes (Fig. 2). The focus of this paper is on the magnetic entropy of noninteracting nanoparticles and of nanoparticles coupled by exchange interactions, with particular emphasis on anisotropy and particle-size dependence.

The equilibrium entropy is calculated from the partition function $Z=\Sigma_{i} \exp \left(-E_{i} / k_{B} T\right)$, where the summation includes all states that are actually accessed during a typical experi-
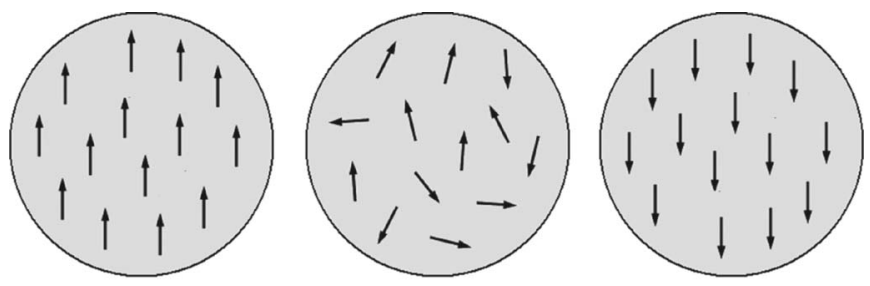

(a)
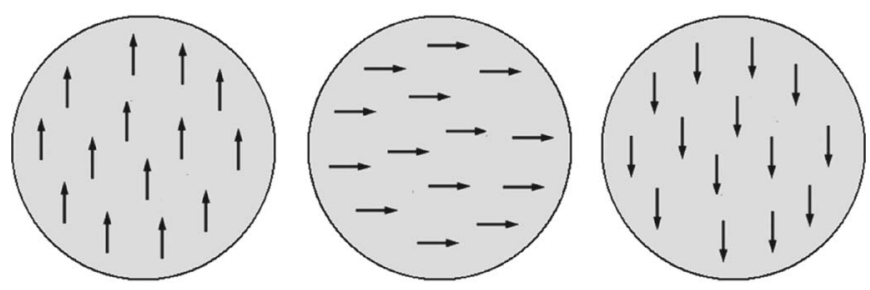

(b)

FIG. 1. Magnetization changes in a nanoparticle: (a) phase transition and (b) magnetization reversal. The change is usually of the type (b), meaning that the relative spin orientation inside the particle is frozen and does not contribute to the entropy change. Each arrow represents an atomic spin. 

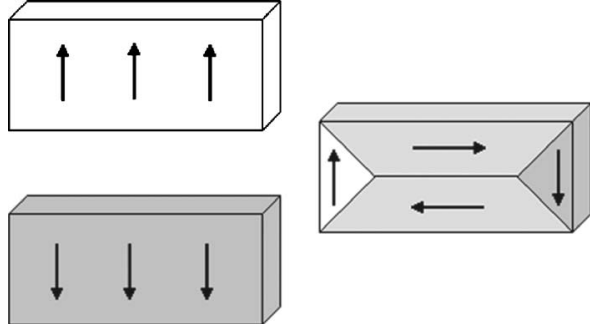

$$
S=k_{B} \ln (2)
$$

(a)

FIG. 2. Realization of states with zero net magnetization: (a) thermodynamic fluctuations and (b) micromagnetic regime.

ment or application cycle. In other words, equilibrium thermodynamics requires ergodicity, but relative magnetization directions are often frozen in magnetic nanostructures and thermodynamic equilibrium is usually limited to length scales of less than about $1 \mathrm{~nm} .{ }^{7,8}$ A similar situation is encountered in mechanical glasses, which are nonergodic because ensemble averages (liquidlike snapshots of pieces of glass) differ from time averages (solidlike frozen atomic configurations). ${ }^{9}$

Thermal averages are obtained from the well-known relation $\langle A\rangle=\Sigma_{i} p_{i} A_{i}$, where $p_{i}=\exp \left(-E_{i} / k_{B} T\right) / Z$ is the probability of encountering the $i$ th state. This includes the expression $S=-k_{B} \Sigma_{i} p_{i} \ln \left(p_{i}\right)$ for the entropy. Free isotropic particles, as well as isotropic particles interacting in the mean-field approximation, obey $E_{i}=-\mu_{0} \mu_{B} H s_{\mathrm{zi}}$, so that $S$ becomes a function of the magnetization. Furthermore, the entropy of nanoparticles exhibits a pronounced dependence on the magnetic anisotropy. This is in contrast to atomic ferromagnetism, where the anisotropy (less than $1 \mathrm{~K}$ per atom) is much smaller than the exchange (more than $100 \mathrm{~K}$ per atom) and important only in the vicinity of $T_{C}$. Nanoparticle anisotropy energies roughly scale as $N \sim R^{3}$ and are often larger than the interparticle interaction.

In the following, we will describe our magnet by the quasiclassical mean-field Hamiltonian,

$$
\mathrm{H}=-N J_{\mathrm{eff}} m\langle m\rangle-N K_{u} m^{2}-N \mu_{0} \mu_{B} H m,
$$

where $K_{u}$ is the uniaxial anisotropy per spin, ${ }^{6,8} m$ is a dimensionless and normalized magnetization variable, and the exchange $J_{\text {eff }}$ describes the interaction between the particles. Dipolar interactions require more elaborate mean-field Hamiltonians, because they may lead to the formation of columnar structures, but otherwise they affect the entropy in a manner similar to exchange.

Let us first neglect the interaction and compare isotropic magnets with uniaxial magnets of anisotropy constant $K_{u}$ (both easy axis and easy plane). There are two well-known and rather trivial high-temperature limits, namely, isotropic particles $\left(K_{u}=0\right)$ and Ising magnets $\left(K_{u}=\infty\right)$. Isotropic particles have $2 N+1$ Zeeman-split states and $S=k_{B} \ln (2 N+1)$, or $S=k_{B} \ln (2 N)$ in the approximation of Eq. (2), whereas Ising magnetism corresponds to two states $\uparrow$ and $\downarrow$ with equal probability and $S=k_{B} \ln (2)$. For arbitrary fields and temperatures, the free energy

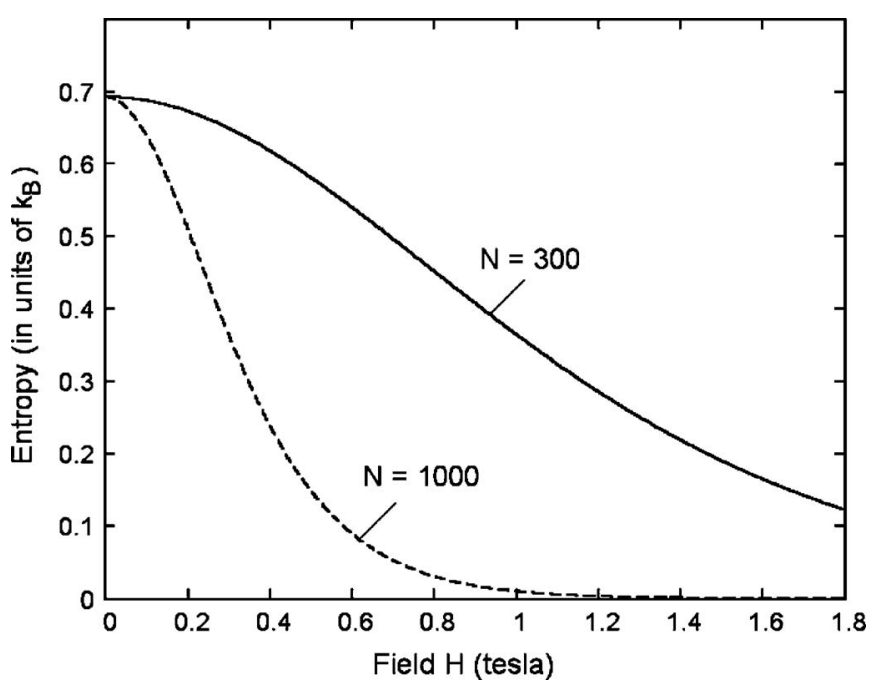

FIG. 3. Entropy of Ising spin clusters.

$$
F=-k_{B} T \ln \left(2 \cosh \frac{\mu_{B} N H}{k_{B} T}\right)
$$

of an Ising spin yields the well-known equation of state $M$ $=\mu_{B} \tanh \left(\mu_{B} N H / k_{B} T\right)$ and the entropy

$$
S=k_{B} \ln \left(2 \cosh \frac{\mu_{B} N H}{k_{B} T}\right)-\frac{\mu_{B} N H}{T} \tanh \frac{\mu_{B} N H}{k_{B} T} .
$$

This entropy decreases from $S=k_{B} \ln 2-\mu_{B}^{2} N^{2} H^{2} / 2 k_{B} T^{2}$ in small fields to $S=0$ in large fields. Fig. 3 shows the roomtemperature entropy for two particle sizes.

The calculation of the partition function for intermediate anisotropy ${ }^{8}$ requires some bookkeeping but is straightforward. Figure 4 shows the room-temperature entropy of noninteracting particles as a function of the anisotropy field $H_{a}$ $=2 K_{u} V_{\text {at }} / \mu_{0} \mu_{B}$, where $V_{\text {at }}$ is the volume per spin. Fields between 0 and about $1 \mathrm{~T}$ are easily created by permanent magnets, and the corresponding entropy gain corresponds to gray area in Fig. 2. We see that isotropic magnets $\left(H_{a}=0\right)$ and

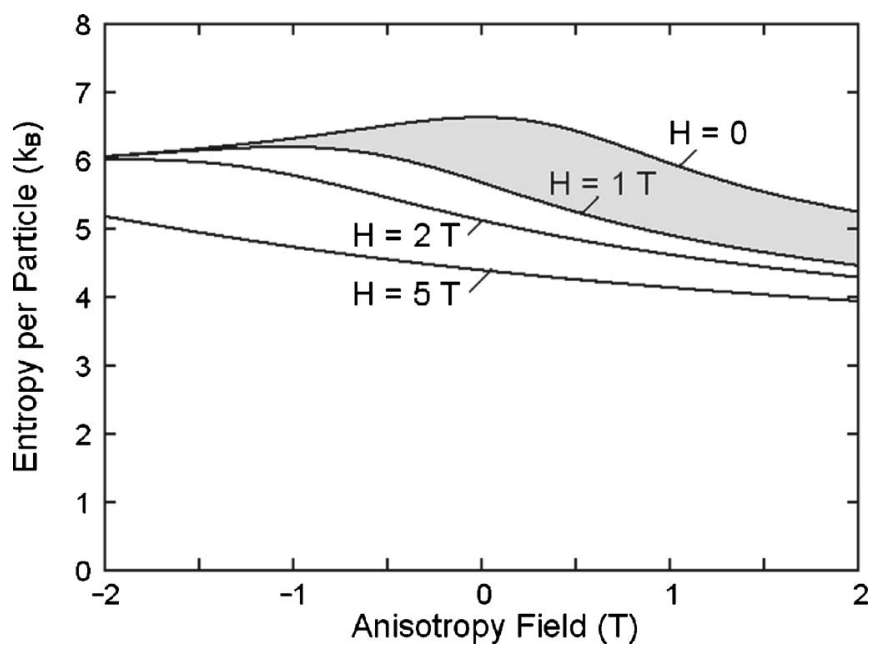

FIG. 4. Field-dependent room-temperature entropy of nanoparticles containing 1000 atomic spins. Negative, zero, and positive anisotropy fields correspond to easy-plane anisotropy, isotropic magnetism, and easy-axis magnetism. The gray area is the field range easily created in permanent-magnet systems. 


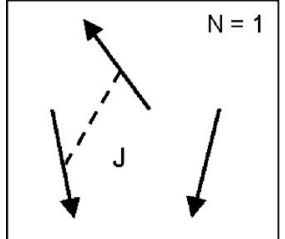

(a)

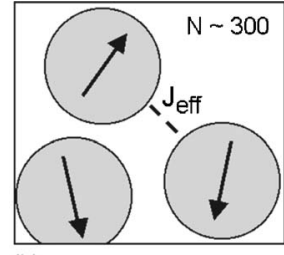

(b)
FIG. 5. Microscopic origin of the entropy change responsible for magnetic cooling: (a) atomic spins in solid and (b) interacting nanoparticles.

magnets with weak easy-axis anisotropy $\left(H_{a}>0\right)$ yield the largest entropy changes. Easy-plane anisotropy $\left(H_{a}<0\right)$ makes the entropy nearly independent of the field component parallel to the anisotropy axis, because the magnetization is largely confined to the basal plane. However, in-plane fields can be shown to yield appreciable entropies.

As in atomically structured magnets, interactions $\left(J_{\text {eff }}\right)$ are potentially helpful to enhance the entropy differences (Fig. 5). The mean-field entropy is obtained by adding the corresponding $J_{\text {eff }}$ term to $H$ in Eq. (4). Figure 6 compares the entropy of interacting and noninteracting particles. The effect is maximized when the interaction strength $J_{\text {eff }} / k_{B}$ is close to room temperature, as assumed for the solid line in Fig. 6. Very small interactions do not yield an appreciable effect, whereas very strong coupling assimilates the system's behavior to macroscopic bulk magnets. However, the finetuning of the interaction is not very critical, because the maximum of the entropy density change is very broad. In small fields, the entropy change for the interacting nanoparticles $\left(J_{\text {eff }}=k_{B} T\right)$ is $\frac{3}{4}\left(\mu_{0} \mu_{B} H / k_{B} T\right)^{4 / 3} N^{1 / 3}$, as compared to the term $\frac{1}{2}\left(\mu_{0} \mu_{B} H / k_{B} T\right)^{2} N$ briefly discussed below Eq. (4).

The maximum entropy of a particle containing $N$ atoms of spin $1 / 2$ is $\ln (2 N)$, so that the entropy per atom is $\ln (2 N) / N$. This entropy scales as $1 / N$, and there is no point

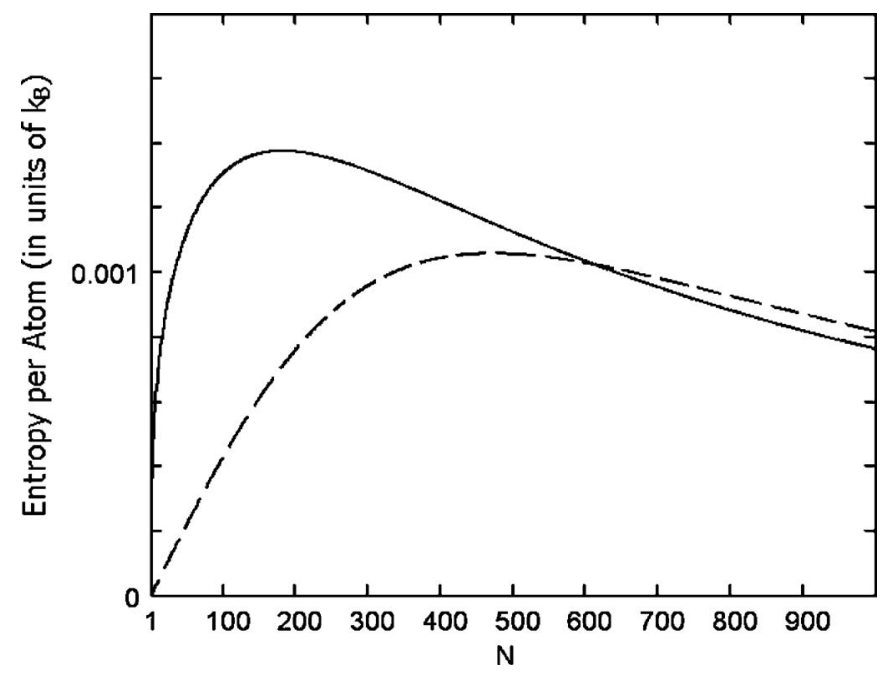

FIG. 6. Room-temperature entropy gain per atom: interacting particles (solid line) and noninteracting particles (dashed line) in a field of $1 \mathrm{~T}$. The interaction is treated on a mean-field level and adjusted to maximize the effect near room temperature.

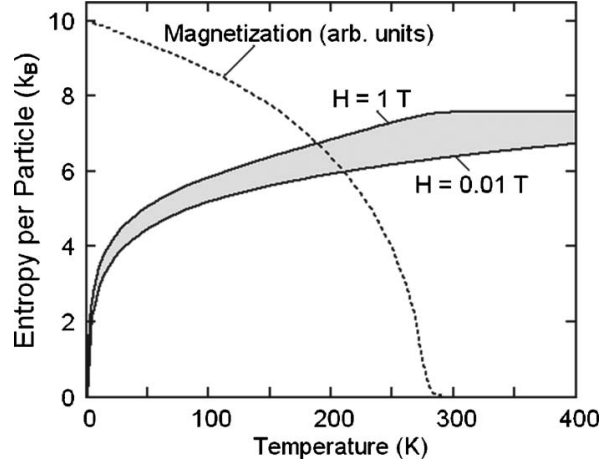

FIG. 7. Temperature dependence of the entropy of nanoparticles having 1000 spins, a net interparticle exchange of $1150 \mathrm{~K}$, and an anisotropy field of $0.1 \mathrm{~T}$. Magnetic cooling exploits the gray area.

in using very large particles. Similar entropy dependences are obtained for other spin models. Since $\mu_{B} / k_{B}$ $=0.672 \mathrm{~K} / \mathrm{T}$, a few hundred spins are sufficient for roomtemperature fields of order $1 \mathrm{~T}$ (Fig. 7), and smaller numbers are achievable by exploiting interactions. We actually promote a hybrid technology intermediate between atomic-scale and nanoscale cooling, combining the benefits of both approaches. Possible practical realizations are embedded nanoparticles weakly interacting through RKKY-type exchange $^{10,11}$ and multilayered magnets with competing ferro- and antiferromagnetic interactions. ${ }^{12}$

In conclusion, we have investigated the entropy gain and magnetic-cooling capabilities of nanostructures for magnetic refrigeration. Nanostructuring improves the entropy change in low fields and at high temperatures but yields some reduction in the maximum entropy change per atom due to frozen magnetization directions in the nanoparticle. An important feature of magnetic nanoparticles is that the entropy exhibits a strong dependence on the magnetic anisotropy. This can be exploited for magnetic cooling.

This work has been supported by NCESR and, partially, by NSF-MRSEC, DOE, and NCMN.

${ }^{1}$ A. L. Lima, K. A. Gschneidner, and V. K. Pecharsky, J. Appl. Phys. 96, 2164 (2004)

${ }^{2}$ V. Provenzano, A. J. Shapiro, and R. D. Shull, Nature (London) 429, 853 (2004).

${ }^{3}$ D. Rebar, J. Gass, P. Poddar, and H. Srikanth, APS March Meeting N42 11, [Bull. Am. Phys. Soc. 50, 912 (2005)].

${ }^{4}$ R. D. McMichael, R. D. Shull, L. J. Swartzendruber, and L. H. Bennett, J. Magn. Magn. Mater. 111, 29 (1992).

${ }^{5}$ S. Mørup, Hyperfine Interact. 90, 171 (1994).

${ }^{6}$ R. Skomski and J. M. D. Coey, Permanent Magnetism (Institute of Physics, Bristol, 1999).

${ }^{7}$ R. Skomski, J. Phys.: Condens. Matter 15, R841 (2003).

${ }^{8}$ R. Skomski, J. Appl. Phys. 83, 6724 (1998).

${ }^{9}$ E.-J. Donth, Glasübergang (Akademie-Verlag, Berlin, 1981).

${ }^{10}$ R. Skomski, Europhys. Lett. 48, 455 (1999).

${ }^{11}$ Advanced Magnetic Nanostructures, edited by D. J. Sellmyer and R. Skomski (Springer, Berlin, 2006).

${ }^{12} \mathrm{Ch}$. Binek, Ising-Type Antiferromagnets: Model Systems in Statistical Physics and in the Magnetism of Exchange Bias (Springer, Berlin, 2003). 